\title{
Política de vivienda y habitabilidad en la periferia de la Zona Metropolitana del Valle de México'
}

\author{
Social housing and habitability in the periphery of \\ Metropolitan Area of the Valley of Mexico
}

\author{
Luis Alberto Salinas Arreortua² (1) y Ana Melisa Pardo Montaño³ (B)
}

\begin{abstract}
RESUMEN
La política de vivienda en México se ha caracterizado por favorecer el acceso en propiedad. Hacia la década de los noventa se promueve la disminución de la intervención del Estado, propiciando el dominio del sector privado en el mercado formal de vivienda. Esto ha incentivado en las ciudades grandes y medias un marcado crecimiento de la oferta de vivienda, tal como ocurre en la periferia oriente y norte de la Zona Metropolitana del Valle de México. En el presente artículo se analizan las características de la oferta de vivienda social a partir del concepto de habitabilidad, contrastando los datos oficiales sobre el mejoramiento de las características físicas de la vivienda con información obtenida a partir de trabajo de campo sobre las condiciones de la vivienda y su entorno urbano. Se plantea que la creciente oferta de vivienda social en la periferia de la Ciudad de México está afectando las condiciones de habitabilidad de los residentes, contrario al discurso oficial de mejoramiento de las condiciones de vivienda.
\end{abstract}

Palabras clave: política de vivienda, vivienda social, habitabilidad, entorno urbano.

\begin{abstract}
The housing policy in Mexico has been characterized by favoring access to property. Towards the decade of the nineties of the twentieth century, the reduction of state intervention is promoted, promoting the domination of the private sector in the formal housing market. This has encouraged large and medium-sized cities with a marked growth in housing supply, as occurs in the eastern and northern periphery of the Metropolitan Area of Valley of Mexico. This article analyzes the characteristics of the social housing based on the concept of habitability, contrasting the official data on the improvement of the physical characteristics of the house with information obtained from field work on the housing conditions and its urban environment. It is suggested that the growing supply of social housing on the periphery of Mexico City is affecting the habitability conditions of the residents, contrary to the official discourse on improving housing conditions.
\end{abstract}

Key words: social housing, habitability, urban environment.

Investigación patrocinada por UNAM - DGAPA. PAPIIT - IN301420 "Sector inmobiliario y gestión urbana en los procesos de revalorización de áreas centrales y expansión de la periferia de la ZMVM".

Instituto de Geografía, Universidad Nacional Autónoma de México. Email: Isalinas@comunidad.unam.mx ORCiD 0000-0002-0983-3629 Instituto de Geografía, Universidad Nacional Autónoma de México. Email: melisa.pardo@comunidad.unam.mx ORCiD 0000-0001-5959-530x 


\section{Introducción}

La política de vivienda en México se ha caracterizado por favorecer el acceso a la vivienda en propiedad desde principios de la década de los setenta del siglo XX, mediante el surgimiento de Organismos Nacionales de Vivienda (ONAVIS), quienes han implementado un sistema de subsidio a la demanda. La intervención del Estado en este sector, denominada "promoción pública de vivienda", incluía desde el acceso al suelo, el control de la producción (contratando a constructoras de vivienda) y su comercialización. Sin embargo, en la década de los noventa se aprecian modificaciones en materia habitacional, que pueden ser consideradas como la disminución de la promoción pública y el dominio de la promoción privada de vivienda. Esto ha incentivado en las ciudades grandes y medias un marcado crecimiento de las periferias urbanas a partir de la oferta de vivienda social ${ }^{4}$ (De Mattos, 2010).

El acelerado crecimiento urbano de la Zona Metropolitana del Valle de México (ZMVM) hacia mediados del siglo XX, fue producto de asentamientos informales, de manera que actualmente más de la mitad del espacio urbano habitacional tiene orígenes en estos asentamientos (Coulomb y Schteingart, 2006; Roberts, 2005; Ward, 2004). Hacia la década de 1970, el subsidio a la demanda implementado por los ONAVIS tiene dos objetivos primordiales: solventar el problema del rezago habitacional y hacer frente al crecimiento de los asentamientos informales. De manera que la continua expansión urbana, y de manera particular en las últimas tres décadas, está ligado al mercado formal de vivienda social, promovida por el sector privado. Este subsidio a la demanda, constituye un mecanismo de la política de vivienda que se ha implementado en diversos países en América Latina, el cual ha incidido en la segregación residencial (Fique, 2008; Zunino e Hidalgo, 2009; Marmolejo, y Batista, 2011; Monkkonen, 2012; Montejano, Caudillo y Cervantes, 2018.). Por segregación residencial se entiende el grado de proximidad espacial o de aglomeración territorial de las familias pertenecientes a un mismo grupo social (Sabatini, Cáceres y Cerda, 2001).

La segregación residencial, para el caso de la ZMVM, se caracteriza por la concentración en la periferia oriente y norte de vivienda social en conjuntos habitacionales, los cuales son proyectos con dimensiones que pueden incluir desde 100 viviendas o más de 25,000. Tal es el caso del conjunto habitacional San Buenaventura, localizado en el municipio de Ixtapaluca, Estado de México. Esta situación tiene una estrecha vinculación a dos factores: por un lado, la política de vivienda establece objetivos cuantificables, es decir, más que una medida de desarrollo urbano que contemple la vivienda y el desarrollo de equipamiento e infraestructura, se concentró únicamente en generar las condiciones para la construcción de vivienda, mediante el fortalecimiento del subsidio a la demanda, con la intención de otorgar un determinado número de créditos hipotecarios; por otro lado, el contexto empresarialista de los gobiernos locales (Harvey, 1989 y 2001), siendo los responsables de la planificación de desarrollo urbano de sus municipios, modifican el uso de suelo según convenga a sus intereses, puesto que la dinámica inmobiliaria favorece el incremento de los ingresos, principalmente mediante el impuesto predial (Salinas, 2016).

\footnotetext{
La Comisión Nacional de Vivienda clasifica a la vivienda según su valor en: Económica (Menos de 118 VSMM = 15,359 dólares). Popular (De 118 VSMM a 200 VSMM = 15,359 a 23999 dólares). Tradicional (De 200 VSMM a 350 VSMM = 23999 a 41998 dólares). Media-Residencial: Incluye vivienda Media, Residencial y Residencial Plus (Más de 350 VSMM = más de 41998 dólares). En este artículo se entenderá vivienda social como vivienda económica.

5 Según el Programa Nacional de Vivienda 2007- 2012: hacia un desarrollo habitacional sustentable, elaborado por la Comisión Nacional de Vivienda establecía la "necesidad" de otorgar 6 millones de financiamientos durante el sexenio 2007- 2012.
} 
Las modificaciones de la política habitacional se han caracterizado por estimular la construcción de vivienda, lo que Connolly (2006) llamó "política de construcción", en el entendido que la construcción de vivienda no estuvo acompañada de desarrollo de infraestructura y equipamiento urbano, y se desestimó la conveniencia de generación de empleo, inversión en educación y salud. Esta forma de favorecer únicamente la construcción de vivienda puede ser parte de los factores que han incidido en las características de habitabilidad. Por habitabilidad se entiende, no sólo las características físicas de la vivienda (diseño, materiales de construcción, metros construidos, superficie habitable), sino, además, el espacio construido entorno a la vivienda, es decir, en relación con las características de localización, proximidad con el empleo, equipamientos educativos, de salud y recreativos, así como la calidad de estos (Coulomb, 2006; Ziccardi, 2015; Salas, 2008; Zulaica y Celemín, 2008).

La política de vivienda que promovió la construcción masiva en periferias urbanas, incidió en procesos de segregación residencial, afectando las condiciones de habitabilidad, lo que repercutió en el abandono de viviendas (Peña y Sandoval, 2017; Cabrera y Guillén, 2018). Sin embargo, desde la administración pública, los diversos instrumentos normativos operacionalizan la medición de las condiciones de habitabilidad con referencia al espacio construido, es decir, a las condiciones físicas e internas de la vivienda, de manera que se ha venido construyendo y reproduciendo un discurso oficial, el cual considera que el esquema del mercado formal de vivienda implementado a principios del presente siglo ha sido todo un éxito ${ }^{6}$. Este es uno de los aportes del presente artículo, en tanto que muchos de los trabajos que analizan las condiciones de habitabilidad se centran en generación de metodologías (Zulaica y Celemín, 2008; Gazmuri, 2013), análisis de casos (Arcas, et. al., 2011; Castillo y Alejandre, 2012; Mejía, 2012; Nascimento, et. al., 2017) o refieren a una discusión de conceptos tales como Justicia espacial (Rincón, 2018), cohesión social (Salas, 2008; Tocornal, et. al., 2014) o seguridad (Capron, 2017) por mencionar algunos ejemplos.

El objetivo del presente artículo es analizar las características de la oferta masiva de vivienda social, a partir del concepto de habitabilidad, en municipios de la ZMVM que han presentado una importante concentración en las últimas dos décadas. Se establece que la creciente oferta de vivienda social en la periferia de la ZMVM forma parte de las acciones de gestionar la vivienda como una mercancía, la cual está determinada por la dinámica del sector inmobiliario, otorgando poca importancia a las condiciones de habitabilidad producidas. De esta manera, se pretende contrastar las condiciones de habitabilidad ofrecidas por la construcción masiva de vivienda, consideradas como un éxito en términos del mercado inmobiliario, con las condiciones de la vivienda y del espacio construido que son valoradas por los residentes de los conjuntos habitacionales. Esto como parte de procesos de segregación residencial promovidos por la política de vivienda.

El presente trabajo contempla información del Instituto del Fondo Nacional de la Vivienda para los Trabajadores (INFONAVIT), Secretaría de Desarrollo Urbano y Metropolitano del Estado de México, la cual es la dependencia responsable de la autorización de los conjuntos habitacionales; censos y conteos de población realizados por el Instituto Nacional de Estadística y Geografía (INEGI), así como del Consejo Nacional de Evaluación de la Política de Desarrollo Social (CO-

El Real Estate Market \& Lifestyle (sitio en internet especializado en información del mercado inmobiliario) menciona que, en materia inmobiliaria, uno de los grandes logros de la administración federal del expresidente mexicano Vicente Fox fue el mercado formal de vivienda, modelo que dio el expresidente Felipe Calderón (2007-2012). 
NEVAL). Se revisaron documentos oficiales del gobierno federal que establecen las condiciones mínimas de habitabilidad, lo que ha favorecido la creciente dinámica del mercado inmobiliario. Por otra parte, para contraponer los discursos que establecen el éxito del mercado inmobiliario habitacional en las periferias urbanas, con las condiciones de habitabilidad generadas, se realizaron entrevistas semiestructuradas a residentes de tres conjuntos habitacionales, localizados en los municipios de la ZMVM que presentan la mayor cantidad de construcción de vivienda social desde el año 2000; Santa Teresa, en Huehuetoca, Paseos de San Juan, en Zumpango y Los Héroes, en Tecámac.

El artículo se organiza de la siguiente manera. Después de la introducción se analizan las características de la construcción masiva de vivienda social en la ZMVM, desde el año 2000 hasta el año 2015, destacando los municipios de Huehuetoca, Tecámac y Zumpango, los cuales han presentado la mayor cantidad de viviendas construidas. En la segunda sección se revisa las discusiones en torno al concepto de habitabilidad. Posteriormente se analiza el uso de conceptos para hacer referencia a calidad de la vivienda por parte de instrumentos normativos de la política habitacional. Mientras que la cuarta sección se contrasta los datos oficiales con las condiciones de habitabilidad mostradas a partir del trabajo de campo. Como parte de las reflexiones se establece un evidente fracaso en materia de integración y desarrollo urbano, pero un éxito en el mercado inmobiliario apoyado por los ONAVIS y justificado por las mediciones de instituciones públicas.

\section{Crecimiento de la ZMVM 2000 - 2015}

La ZMVM está conformada por 16 alcaldías de la Ciudad de México y 60 municipios (59 del Estado de México y uno del estado de Hidalgo), dando un total de 76 unidades territoriales. En los municipios del estado de México e Hidalgo que forman parte de la zona metropolitana, se autorizaron la construcción de 687,263 viviendas durante 2000 a 2015, siendo Huehuetoca, Tecámac y Zumpango tres de los municipios metropolitanos con el mayor número de viviendas autorizadas para su construcción, los cuales registraron 278,474 viviendas, es decir, en estos tres municipios se concentra el $40.51 \%$ de la oferta total de viviendas de los municipios que integran la ZMVM (ver figura 1). Este crecimiento de oferta de vivienda incide en que estos tres municipios registren las tasas más altas de crecimiento poblacional.

En Huehuetoca se incrementó la población de 38,458 a 128,486 habitantes de 2000 a 2015, un aumento que representa el 234\%. Tecámac pasó de 172,813 a 446,008 habitantes en el mismo periodo, mostrando un crecimiento del 158\%. Mientras que Zumpango pasó de 99,774 a 199,069 habitantes, representando un $100 \%$ de incremento. En los tres municipios los porcentajes de aumento están muy por encima del registrado a nivel estatal el cual fue del $24 \%$. La tasa media de crecimiento anual en este mismo periodo fue de $8.37 \%$ en Huehuetoca, $6.52 \%$ en Tecámac y $4.71 \%$ en Zumpango, mientras que en el estado de México fue de tan sólo 1.42\% (INEGI, 2015).

A pesar de este fuerte incremento de población, todavía se encuentra por debajo de las proyecciones esperadas por el gobierno del estado de México, derivado de la cantidad de oferta de vivienda en estos municipios. Según las estimaciones de la autorización de los conjuntos habitacionales, se preveía que, para el caso de Huehuetoca, el incremento de 77,484 viviendas beneficiaría a una población estimada en 348,678; mientras que, en Tecámac, la autorización de 
Figura 1. Oferta de vivienda en la ZMVM, 2000 - 2015.
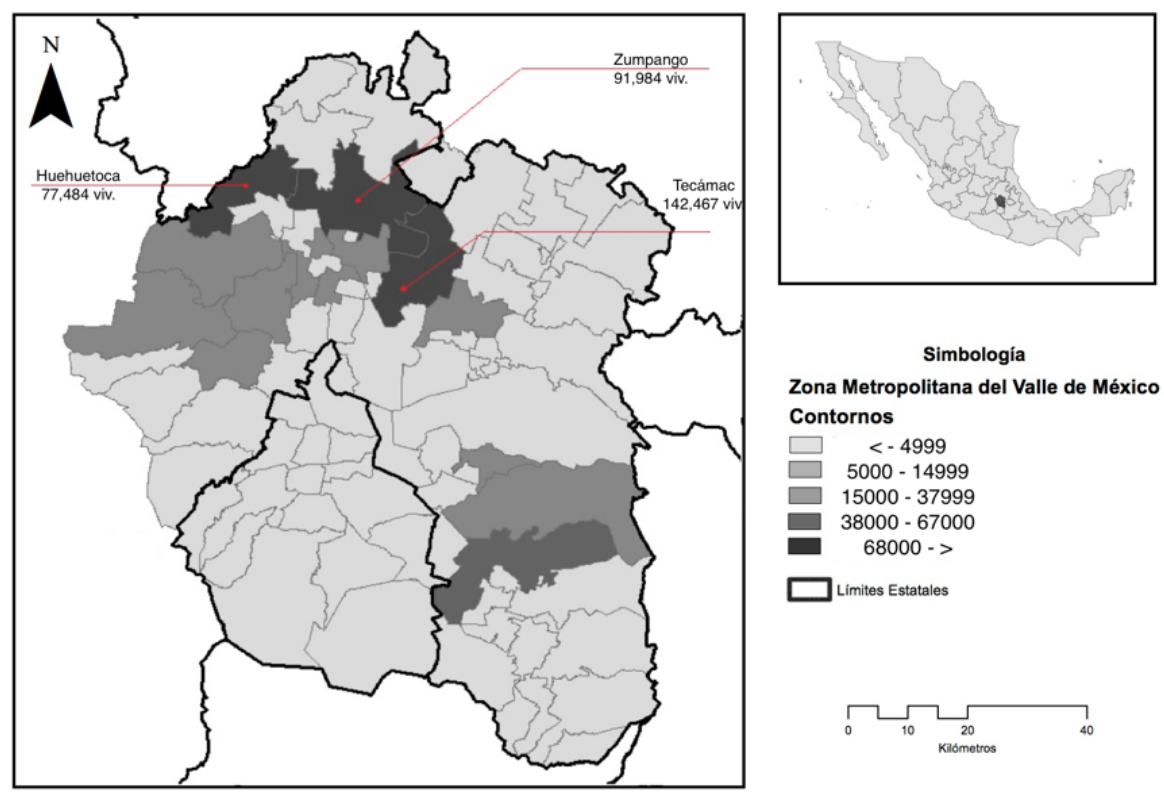

Zona Metro
Contornos

$\square<-4999$

$5000-14999$

$15000-37999$

$38000-67000$

$68000->$

Limites Estatales

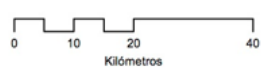

Fuente: Elaboración propia con información de la Secretaría de Desarrollo Urbano y Metropolitano del Estado de México.

142,467 viviendas beneficiaría a 677,720 habitantes; y Zumpango, se autorizaron 91,984 viviendas para una población de 418,249. Las estimaciones contemplan que cada vivienda beneficiaría a 4.5 personas aproximadamente. No obstante, dicha situación se debe, entre otros factores, a una importante presencia de viviendas deshabitadas y abandonadas ${ }^{7}$. Según el INFONAVIT (2015), el estado de México ocupa el 4to lugar en vivienda abandonada y la Ciudad de México el 7mo lugar, mientras que, por zonas metropolitanas, la ZMVM ocupa el primer lugar con 38,093 viviendas abandonadas, le siguen la zona metropolitana de Monterrey con 19,780 y la zona metropolitana de Guadalajara con 18,347.

Un factor que incide en el abandono de vivienda es la localización periférica, en relación con las actividades por las que se vinculan los residentes con las áreas centrales de la Ciudad de México, es decir, principalmente por carencia de empleo, servicios educativos y de salud (INFONAVIT, 2015). Sin embargo, según el trabajo de campo realizado en diversos conjuntos habitacionales, para el caso de municipios como Huehuetoca y Zumpango, también ha incidido que las inmobiliarias no hayan cumplido a cabalidad con el proyecto autorizado, de manera que no ha sido posible

Se entiende por vivienda deshabitada aquella que durante las visitas domiciliarias para el levantamiento censal realizado por el INEGI, no reportaron explícitamente tener habitantes, es decir, según la apariencia y confirmación de los vecinos, no hay evidencia de que alguien viva en dicha casa, lo cual no implica necesariamente que la vivienda esté en malas condiciones (pues claramente existen viviendas nuevas que aún no están habitadas). Mientras que una vivienda abandonada, según el INFONAVIT, es aquella que ha dejado de pagar el derechoabiente (INFONAVIT, 2015). 
el proceso de entrega-recepción ante las autoridades municipales, razón por la cual algunos conjuntos habitacionales carecen de los servicios básicos.

\section{Características de habitabilidad en la periferia urbana}

Sobre la definición de habitabilidad no existe consenso en la academia. Suele definirse en función de las características físicas de construcción, de la superficie habitable, la disponibilidad de infraestructura sanitaria, entre otras. Pero también resulta importante definirla con relación a las cualidades del entorno de la vivienda, localización, proximidad o lejanía con el empleo (tiempo y costo del traslado vivienda-trabajo), los equipamientos educativos, de salud y recreativos, espacios abiertos de encuentro y convivencia, así como la calidad de estos (Gramsch, 2006; Castillo y Alejandre, 2012; Mellado, 2015).

Además de las características físicas de la vivienda y de su localización, también es común considerar aspectos subjetivos. Moreno (2008), basándose en la pirámide de necesidades de Maslow, establece que la habitabilidad está determinada por la relación y adecuación entre el hombre y su entorno, y se refiere a cómo cada una de las escalas territoriales es evaluada según su capacidad de satisfacer las necesidades humanas. En este mismo sentido, Lándazuri y Mercado (2004), afirman que la habitabilidad "es el atributo de los espacios construidos de satisfacer las necesidades objetivas y subjetivas de los individuos y grupos que las ocupan, es decir, las esferas psíquicas y sociales de la existencia estable que podría equipararse a las cualidades medioambientales que permitan el sano desarrollo físico, biológico, psicológico y social de la persona." (Lándazuri y Mercado, 2004:90).

Ceballos (2006) y Ziccardi (2015) establecen dos dimensiones fundamentales para aproximarse al análisis de la habitabilidad: la física y la social. En la primera, se tienen el factor urbanístico, con su atributo localización, y el factor arquitectónico, con sus atributos higiene, privacidad, comodidad y protección, es decir, las características internas. En la segunda, la dimensión social, se contemplan las condiciones de adecuación social y de seguridad en la tenencia, es decir, características externas. Establecen así factores tanto objetivos como subjetivos relacionados con la calidad de la vivienda. Aunado a lo anterior, destaca una preocupación a tenerse en cuenta, los estudios relacionados con el análisis de calidad de la vivienda o bien apuntaban a factores objetivos o bien a los factores subjetivos, sin revisar ambos de manera simultánea.

Mientras que Gazmuri (2013) establece la "habitabilidad mínima" o básica, la cual debe incluir infraestructura y servicios primarios a nivel de comunidad o recinto vecinal, tales como abastecimiento de agua potable, saneamiento, eliminación de desechos, transporte y comunicaciones, energía, entre otros.

De esta manera, se tiende a establecer las condiciones de habitabilidad en dos dimensiones: características internas y externas (Coulomb, 2006; Gramsch, 2006; Castillo y Alejandre, 2012; Mellado, 2015; Ziccardi, 2015); características físicas y sociales (Ceballos, 2006). Por otra parte, también se vincula la habitabilidad con la satisfacción de la vivienda, considerando aspectos objetivos como subjetivos (Moreno, 2008; Lándazuri y Mercado, 2004). 
Por habitabilidad será importante considerar tanto los aspectos físicos de la vivienda, así como del espacio construido, en donde no basta con la existencia de infraestructura, servicios y equipamiento sino con la calidad de estos. Retomando la definición propuesta por Coulomb establece que "...la habitabilidad de una vivienda está entonces en función no sólo de la calidad de sus materiales constructivos, de la superficie habitable o de la disponibilidad de los servicios de agua y saneamiento. Está en relación también con la proximidad o lejanía con el empleo (tiempo y costo del traslado vivienda-trabajo), los equipamientos educativos, de salud y recreativos, de la calidad de los espacios abiertos de encuentro y convivencia, etcétera." (2006:36). Más aún, para Capron (2017) además de la calidad del espacio físico, sea de la vivienda y de las condiciones "externas" (proximidad al empleo, equipamientos y servicios), la habitabilidad se relaciona a la calidad de las relaciones sociales, individuales y colectivas, que los individuos mantienen entre sí. Por lo tanto, la oferta de vivienda debería considerar estos elementos para generar mejores condiciones de habitabilidad, y no sólo gestionar la vivienda como una mercancía, sino como un derecho humano y como un espacio para la reproducción social.

Las características de habitabilidad, que se traducen más en condiciones espaciales - calidad de las relaciones sociales, individuales y colectivas (Capron, 2017) - que en la estructura física de la vivienda, pueden mantener cierta relación con elementos que caracterizan la segregación residencial. Marmolejo y Batista (2011) en un interesante análisis para el caso de ciudades brasileñas, establecen justamente que las zonas con servicios urbanos aceptables poseen la mayor diversidad de grupos socioeconómicos, por el contrario, las zonas marginadas de dichos servicios no sólo están poco diversificadas, sino también, son el escenario en el cual se potencian los fenómenos de segregación. De esta manera, coincidiendo con el análisis mencionado, una de las características de los conjuntos habitacionales de la periferia norte de la ZMVM, es que están destinados a población de bajos ingresos, quienes mediante un crédito público acceden a una vivienda en condiciones de escasez de infraestructura, equipamiento y servicios, reproduciendo de manera paralela problemáticas relacionadas a localización lejana a centralidades urbanas, principalmente al empleo.

\section{Concepto de habitabilidad en la política habitacional}

El concepto habitabilidad ha sido muy discutido en el ámbito académico, mientras que en el sector público es tratado sólo en algunas disposiciones normativas. Para hacer referencia a condiciones de la vivienda el gobierno federal suele utilizar otros conceptos. En la Constitución política de los Estados Unidos Mexicanos, en el artículo $4^{\circ}$ establece el derecho de toda familia a disponer de una "vivienda digna y decorosa". La ley secundaria competente, Ley de Vivienda (2006), en el artículo $2^{\circ}$ la define como:

"Se considerará vivienda digna y decorosa la que cumpla con las disposiciones jurídicas aplicables en materia de asentamientos humanos y construcción, salubridad, cuente con espacios habitables y auxiliares, así como con los servicios básicos y brinde a sus ocupantes seguridad jurídica en cuanto a su propiedad o legitima posesión, y contemple criterios para la prevención de desastres y la protección física de sus ocupantes ante los elementos naturales potencialmente agresivos." 
En atención a este artículo de la Ley de Vivienda, la Comisión Nacional de Vivienda (CONAVI) publicó el Código de Edificación de Vivienda (CEV), el cual es un documento que rige la edificación en el ámbito nacional. Define la "vivienda digna" como:

"Vivienda digna: es considerado como el límite inferior al que se pueden reducir las características de la vivienda sin sacrificar su eficacia como satisfactor de las necesidades básicas, no suntuarias, habitacionales de sus ocupantes. Este tipo de vivienda cumpliría simultáneamente con los siguientes requisitos: a) estar ocupada por una familia, b) no tener más de 2.5 habitantes por cuarto habitable; c) no estar deteriorada, d) contar con agua entubada en el interior, e) contar con drenaje; f) contar con energía eléctrica. Adicionalmente, la vivienda debe proveer entre otras, las siguientes condiciones: protección, para aislar en forma suficiente, permanente y regulable a voluntad, de los agentes exteriores potencialmente agresivos, de origen climático, residual, de catástrofes naturales, etc.; condiciones de higiene suficientes para reducir enfermedades patógenas imputables a las características de la casa habitación, tales como: ventilación, asoleamiento e iluminación, espacio útil por ocupante que evite el hacinamiento (proximidad obligada, persistente, interferencia entre los ocupantes de un recinto o vivienda), flexibilidad e idoneidad en las instalaciones para el almacenamiento de agua potable, disposición y eliminación adecuada de residuos. Asimismo, debe permitir privacidad externa e interna, comodidad y funcionalidad mediante un diseño idóneo y uso correcto de los materiales de construcción que propicie la expresión cultural de sus ocupantes; localización adecuada y seguridad en la tenencia." (Código de Edificación de Vivienda, 2010:52).

No obstante, en este mismo código se define habitabilidad como: "las condiciones en las que la familia habita una vivienda: Estas condiciones están determinadas tanto por las características físicas de la vivienda y de sitio, como por las características psicosociales de la familia, que se expresan en hábitos, conductas o maneras de ser adquiridos en el transcurso del tiempo." (Código de Edificación de Vivienda, 2010:318). Importante su definición; sin embargo, es sólo nombrado en el "Glosario de términos", de manera que no forma parte de un instrumento que procure la calidad de la vivienda y el espacio construido.

La CONAVI maneja el concepto de habitabilidad muy restringido, cercano al concepto de vivienda digna. Dando seguimiento a la Ley de vivienda, dicha institución se ha encargado de establecer criterios para determinar las condiciones de la vivienda, teniendo en cuenta dos dimensiones: calidad y espacios de la vivienda y servicios básicos. Del primero se considera como población en situación de carencia por calidad y espacios de la vivienda a las personas que residan en viviendas que presenten, al menos, una de las siguientes características: a) material de los pisos de la vivienda de tierra, b) material del techo de la vivienda de lámina, cartón o desechos, c) material de los muros de la vivienda de embarro o bajareque, carrizo, bambú o palma, lámina de cartón, metálica o asbesto, o material de desecho, y d) personas por cuarto mayor a 2.5 (hacinamiento). Respecto a los servicios básicos con los que debe contar una vivienda se tienen en cuenta: acceso al agua potable, disponibilidad de servicio de drenaje, servicio de electricidad y combustible para cocinar en la vivienda.

Cabe mencionar que esta definición es tomada de un trabajo realizado por Roberto Eibenschutz y Carlos Goya (2009). 
Con base en lo anterior, el Consejo Nacional de Evaluación de la Política de Desarrollo Social (CONEVAL), realizó la medición de la carencia en la calidad y espacios y servicios básicos en la vivienda, a nivel nacional y por entidad federativa, con base en los censos de población y vivienda 1990, 2000 y 2010 desarrollados por el INEGI. De esta manera, se puede apreciar en el cuadro No 1 la disminución del porcentaje de población que carece de calidad y espacios de la vivienda en los últimos años, tanto a nivel nacional como en los estados que forman la ZMVM. Mientras que el cuadro $N^{\circ} 2$ se presenta los servicios básicos. Cabe mencionar que se actualizó la información en ambos cuadros a partir de la Encuesta Intercensal 2015 elaborada también por el INEGI.

Cuadro No 1. Evolución de la carencia por calidad y espacios de la vivienda 1990 - 2015 (\%).

\begin{tabular}{|c|c|c|c|c|c|c|c|c|c|c|c|c|c|c|c|c|}
\hline & \multicolumn{4}{|c|}{ Pisos de tierra } & \multicolumn{4}{c|}{$\begin{array}{c}\text { Techo de material } \\
\text { de desecho }\end{array}$} & \multicolumn{3}{c|}{$\begin{array}{c}\text { Muros de material } \\
\text { de desecho }\end{array}$} & \multicolumn{3}{c|}{ Hacinamiento } \\
\hline & 1990 & 2000 & 2010 & 2015 & 1990 & 2000 & 2010 & 2015 & 1990 & 2000 & 2010 & 2015 & 1990 & 2000 & 2010 & 2015 \\
\hline Huehuetoca & 4.8 & 3.4 & 2.1 & 0.5 & 2.6 & 2.3 & 1.2 & 0.4 & 0.9 & 0.0 & 0.4 & 0.2 & 21.8 & 17.9 & 17.4 & $\mathrm{~s} / \mathrm{d}$ \\
\hline Tecámac & 7.1 & 4.2 & 2.3 & 0.6 & 7.9 & 5.5 & 0.5 & 0.7 & 0.9 & 0.1 & 0.6 & 0.1 & 22.9 & 20.3 & 12.1 & $\mathrm{~s} / \mathrm{d}$ \\
\hline Zumpango & 8.8 & 4.4 & 3.0 & 1.0 & 4.6 & 1.9 & 0.4 & 0.3 & 1.2 & 0.0 & 0.2 & 0.1 & 21.3 & 15.8 & 18.2 & $\mathrm{~s} / \mathrm{d}$ \\
\hline Edo. de Mex. & 11.9 & 6.4 & 3.8 & 1.8 & 10.5 & 6.6 & 3.4 & 2.7 & 1.3 & 0.8 & 0.6 & 0.3 & 23.2 & 18.5 & 10.6 & 9.7 \\
\hline Ciudad de Mex. & 2.1 & 1.2 & 1.0 & 0.4 & 6.3 & 3.4 & 1.5 & 1.0 & 1.2 & 0.9 & 0.5 & 0.3 & 17.3 & 12.9 & 9.3 & 6.9 \\
\hline Nacional & 19.5 & 13.2 & 6.2 & 3.6 & 10.0 & 6.2 & 0.2 & 0.6 & 6.4 & 4.0 & 2.1 & 0.2 & 23.7 & 17.8 & 12.8 & 6.9 \\
\hline
\end{tabular}

Fuente: elaboración propia a partir de información de CONEVAL y de la Encuesta Intercensal, INEGI, 2015.

Cuadro No 2. Evolución de la cobertura de servicios básicos 1990 - 2015

\begin{tabular}{|c|c|c|c|c|c|c|c|c|c|c|c|c|c|c|c|c|}
\hline & \multicolumn{3}{|c|}{ Distribución de agua } & \multicolumn{3}{|c|}{ Drenaje sanitario } & \multicolumn{3}{c|}{ Energía eléctrica } & \multicolumn{4}{c|}{ Combustible } \\
\hline & 1990 & 2000 & 2010 & 2015 & 1990 & 2000 & 2010 & 2015 & 1990 & 2000 & 2010 & 2015 & 1990 & 2000 & 2010 & 2015 \\
\hline Huehuetoca & 93.2 & 85.3 & 98.7 & 98.4 & 69.4 & 85.4 & 98.7 & 98.2 & 96.2 & 94.1 & 99.5 & 99.5 & 94.6 & 94.7 & 92.3 & 96.3 \\
\hline Tecámac & 88.1 & 90.0 & 98.4 & 98.9 & 78.1 & 89.3 & 99.0 & 98.9 & 97.0 & 93.1 & 99.5 & 99.9 & 93.9 & 93.6 & 98.5 & 98.2 \\
\hline Zumpango & 95.2 & 88.4 & 95.4 & 96.5 & 70.8 & 85.7 & 97.7 & 97.9 & 96.7 & 94.3 & 99.0 & 99.6 & 92.8 & 94.2 & 97.3 & 96.5 \\
\hline Edo. de Mex. & 85.1 & 89.9 & 92.9 & 95.7 & 74.0 & 86.3 & 92.6 & 95.5 & 93.8 & 97.9 & 97.3 & 99.4 & 94.1 & 94.7 & 92.3 & 91.5 \\
\hline Ciudad de Mex. & 96.3 & 96.9 & 95.1 & 98.6 & 93.7 & 98.2 & 96.3 & 98.8 & 99.3 & 99.6 & 96.8 & 99.8 & 93.6 & 95.0 & s/d & 97.1 \\
\hline Nacional & 78.7 & 82.6 & 90.0 & 94.6 & 63.0 & 76.5 & 88.8 & 92.8 & 86.7 & 93.1 & 96.2 & 98.7 & 91.0 & 91.7 & s/d & 93.0 \\
\hline
\end{tabular}

Fuente: elaboración propia a partir de información de CONEVAL, Encuesta Intercensal, INEGI, 2015 y del Sistema Nacional de información Municipal.

De esta forma, según CONEVAL, con información de INEGI, los municipios que integran la ZMVM han mejorado la calidad y espacios de la vivienda y también en cuanto a cobertura de servicios básicos, tal como se muestra en los cuadros 1 y 2 . Se puede apreciar que la medición se centra tanto en condiciones físicas e internas de la vivienda, sin considerar los factores externos.

Aunado a lo anterior, como se mencionó con la definición proporcionada por el CEV y los elementos considerados para la medición de la calidad y espacios de la vivienda y de los servicios básicos por el CONEVAL, las instituciones gubernamentales han operacionalizado el concepto de 
habitabilidad, derivado de vivienda "digna y decorosa" establecido en la constitución, a partir de características físicas e internas de la vivienda, y de manera particular con las condiciones mínimas del espacio habitable, tal como lo establece el CEV. Por ello, es necesario destacar la calidad, en términos de habitabilidad, debido a que ha perdurado una visión cuantitativista que prevalece tanto en el mercado habitacional como en las instituciones gubernamentales encargadas de diseñar e implementar la política habitacional (Arcas, et. al., 2011; Ziccardi, 2015).

Además de las características utilizadas por el gobierno federal para analizar la situación y evolución de la vivienda, los programas desarrollados para mejorar la habitabilidad giran en ese mismo sentido. El Fondo Nacional de Habitaciones Populares (FONHAPO), institución encargada se satisfacer necesidades de vivienda a población de bajos ingresos, ha desarrollado distintos programas para mejorar la vivienda "digna", tal es el caso del Programa Vivienda Digna que otorga subsidios a los hogares en situación de pobreza, carentes de calidad y de espacios de la vivienda adecuados. En este mismo sentido, el Instituto Mexiquense de la Vivienda Social ha implementado un programa para que las familias mexiquenses mejoren las características de "habitabilidad", las cuales se basan en mejorar las condiciones de piso firme, tinaco (grandes recipientes para almacenar agua), letrina seca (espacio en el subsuelo destinado al drenaje, el cual no está conectado a ninguna alcantarilla) y techo de lámina o loza de concreto 9 .

En este orden de ideas, Kunz y Soledad (2017) mencionan que durante el diseño de la política pública de construcción masiva de vivienda, confundieron las metas (número de viviendas) con los objetivos (condición de habitación), evidentemente haciendo referencia a que intencionalmente se ha favorecido la reproducción del capital inmobiliario, pues la construcción de vivienda es considerada más por su importancia en materia económica. En una entrevista con el entonces candidato a la presidencia Felipe Calderón, quien gobernó del año 2007 al 2012, establecía la importancia de la vivienda, más que como un derecho social, como un factor económico: la vivienda es importante "...porque da inversión y el empleo que México necesita, porque la vivienda le da patrimonio a las familias y estabilidad a nuestro país"10.

La política de vivienda en México se ha centrado en la construcción masiva, entendiendo que es parte importante del sector económico, en particular de la industria de la construcción, descuidando las condiciones de reproducción social de los residentes, de esta manera, las condiciones de habitabilidad se han visto mermadas dentro del mercado formal de vivienda, tal como se verá en el siguiente apartado.

\section{Características de habitabilidad en la periferia de la ZMVM}

El presente análisis se desarrolló a partir del trabajo de campo realizado, entre abril y agosto de 2017, el cual consistió en la realización de 81 entrevistas semiestructuradas aplicadas a residentes de tres conjuntos habitacionales: Santa Teresa, en el municipio de Huehuetoca, Los Héroes,

\footnotetext{
Información disponible en el Instituto Mexiquense de la Vivienda Social. http://imevis.edomex.gob.mx/mejoramiento_de_vivienda

Entrevista realizada y publicada por el Real Estate Market \& Lifestyle, 2006.
} 
localizado en el municipio de Tecámac y Paseos San Juan, en el municipio de Zumpango (ver Mapa 1). Se decidió trabajar en estos conjuntos habitacionales debido a que los tres se construyeron en el mismo año (2005), presentan una tipología de vivienda catalogada como mixta: interés social, comercial y de servicios, y fueron construidas por empresas que dominan en cuanto a cantidad de viviendas producidas en cada uno de los municipios; Casas Beta del Centro (Homex), en Huehuetoca, Desarrollos Inmobiliarios Sadasi, para el caso de Tecámac y GEO Hogares ideales, en Zumpango. Estas empresas que conformaron monopolios en cada municipio ofrecen condiciones similares en cada uno de sus conjuntos habitacionales en términos de las características internas y externas de la vivienda, por ello, resultó pertinente elegir estos conjuntos habitacionales como caso de estudio.

Por su parte, las entrevistas se aplicaron a residentes que fueran mayor de edad, que llevaran viviendo más de 10 años, lo que en muchos casos representaba personas propietarias de la vivienda y que accedieron a ella mediante un crédito hipotecario por parte de una institución pública (Fovissste ${ }^{11} \mathrm{o}$ Infonavit). De esta manera, los entrevistados tendrían un panorama general respecto a las condiciones físicas de la vivienda así como a las características externas, en particular con proximidad de empleo, equipamientos y servicios básicos. El análisis de las entrevistas se realizó mediante el software Atlas TI.

La información se organiza de la siguiente manera. En primer lugar se tratan dos temas que hacen referencia directa con las mediciones elaboradas por las instituciones públicas, las cuales son las condiciones físicas de la vivienda (cuadro 1) y los servicios básicos, de manera particular distribución de agua, drenaje sanitario, abastecimiento de energía eléctrica y combustible para cocinar (cuadro 2). Por otra parte, se presenta información sobre equipamiento, espacio público y localización, los cuales son características que no han sido medidas por instituciones públicas pero que son centrales en el concepto de habitabilidad.

Según información censal utilizada por el CONEVAL, el mercado formal de vivienda económica ha mejorado las condiciones físicas e internas en prácticamente todos los rubros considerados. En los municipios en cuestión (Huehuetoca, Tecámac y Zumpango), del total de las viviendas habitadas ha descendido el porcentaje de viviendas con pisos de tierra, techos y muros de material de desecho y hacinamiento (ver cuadro No 1). Así también ha mejorado sus características internas, en lo que se refiere a distribución de agua, drenaje sanitario, abastecimiento de energía eléctrica y combustible para cocinar (ver cuadro $N^{\circ} 2$ ).

\section{Características físicas de la vivienda}

Las características físicas e internas de la vivienda, que han sido medidas por instituciones federales, a pesar de que en los números existe una mejoría, los testimonios de los residentes expresan lo contrario. Esta situación se refleja en la insatisfacción de la vivienda, en términos de sus cualidades físicas mencionadas. Al preguntar sobre las condiciones de la vivienda, los residentes de los tres conjuntos habitacionales, independientemente de la empresa constructora, no están satisfechos:

Fondo de la Vivienda del Instituto de Seguridad y Servicios Sociales de los Trabajadores del Estado. 
"Desgraciadamente, cuando compramos la vivienda, todo estaba perfectamente, pero hay vicios ocultos, que después de determinado tiempo van saliendo. Como son grietas, como son fallas. Lo que si no puedo considerar el material, porque pues no soy ingeniero, para determinarlo. Pero creo yo que los materiales, si tiene uno duda." (Residente de Los Héroes, Tecámac).

"Yo soy Ingeniero Civil, están deficientes totalmente, son unas viviendas hechas como si fueran de deshecho, de reciclaje. Hay materiales que en ocasiones, por ejemplo, haces una perforación para colgar algo, para poner un taquete y está deprimente la forma. De por si es material hueco, son blocks huecos, pero si hablamos de las trabes o las losas luego se siente cuando un material está hecho con resistencia, las resistencias de los materiales no son las adecuadas. No es posible que hagas un taquete y que se desmorone la columna. A lo mejor donde esta hueco porque estas son casas con loza de vigueta y bovedilla, tienen huecos donde van los uniceles, pero es deficiente la calidad. La elaboración, además de los materiales, yo creo que la construcción, el proceso constructivo es malo, ya tenemos muchas fisuras en todos lados, en las salientes que son como marquesinas en las ventanas tienen una boquilla que se están rompiendo en todas las ventanas de todas las casas porque la calidad de los materiales no es la adecuada." (Residente de Paseos de San Juan, Zumpango).

"Bueno de la vivienda es, son los materiales, que es muy aparte de ser muy pequeña o acogedora como no la vendieron es este, de muy mala elaboración porque los materiales que están realizados no son de calidad, no tienen alta calidad o no estaban estipulando que iba a llover, yo creo, porque hay mucha humedad, aparte de la humedad compartimos pared, incluso si la vecina que es doctora, da sus masajes, yo escucho como al señor le duelen los huesos o sea se escucha todo incluso hasta para hablar por teléfono todo se escucha, todo, todo, todo, de verdad, sabemos la vida de todos aunque no queramos, ahh es lunes hoy si toca..." (Residente de Santa Teresa, Huehuetoca).

Como se puede apreciar, en los tres conjuntos habitacionales los residentes entrevistados se quejan por los materiales de construcción. Tanto del material de los techos como de las paredes, en particular porque una pared suele dividir dos viviendas. De manera que, a pesar del discurso oficial basado en las mediciones realizadas por el CONEVAL con información censal, los residentes están insatisfechos con la calidad de los materiales de construcción de su vivienda.

\section{Servicios básicos (distribución de agua, drenaje sanitario, abasteci- miento de energía eléctrica y combustible para cocinar)}

Por otra parte, más allá de las características físicas de la vivienda, los testimonios coinciden en las malas condiciones de los servicios básicos, considerando por estos, la distribución de agua, drenaje sanitario, abastecimiento de energía eléctrica y combustible para cocinar.

Con excepción del drenaje y del combustible, que en el primer caso no se registró testimonios de mal funcionamiento y en el segundo es suministrado por empresas privadas, hay muchos problemas con el abastecimiento de agua potable y la irregularidad en el servicio en la energía eléctrica. Respecto al abastecimiento de agua suele ser común que a determinadas horas durante el día se corta el suministro, llegando a pasar días enteros sin agua, a pesar de que las cifras oficiales mencionan la cobertura de agua entre un $96 \%$ y $98 \%$ (Cuadro $N^{\circ} 2$ ). Por su parte, el $99 \%$ 
de viviendas cuenta con instalaciones eléctricas; sin embargo, las irregularidades se presentan tanto en el corte de suministro de energía eléctrica, así como en variaciones del voltaje, lo que repercute en el funcionamiento de los aparatos electrónicos. Algunos testimonios dan muestra de los problemas con el abastecimiento de agua:

"Si, te digo que nos la ponen a las 5:30 o 6 de la mañana y la quitan a las 10 o las 11 y ya de ahí todo el pinche día te la avientas sin agua, ya hasta como a las 6 o 7 la vuelven a poner y la ponen como hasta las 10, 11 de la noche y la vuelven a quitar y así, es algo tedioso." (Residente de Santa Teresa, Huehuetoca).

"Luego hasta nos la quitan luego dos, tres días. Hubo un tiempo que... como más de seis meses que no más nos echaban en la mañana y un chorrito. Ahorita ya más o menos. Tiene como un mes que ya, ya tenemos un poquito más de agua." (Residente de Santa Teresa, Huehuetoca).

"Pues el agua a veces la quitan, a veces huele muy feo, a veces sale muy sucia... La luz pues a veces se va, así está." (Residente de Los Héroes, Técamac).

Como se puede observar, a pesar de que en las cifras oficiales en estos tres municipios el porcentaje de viviendas que cuentan con cobertura de agua e instalaciones eléctricas es por arriba del promedio del estado de México y a nivel nacional, las condiciones respecto al abastecimiento distan mucho de lo que se muestra en los censos.

\section{Equipamiento, espacio público y localización}

Tanto las condiciones físicas de la vivienda como los servicios básicos mencionados son aspectos que han sido medidos por el CONEVAL con base en información del INEGI. Pero hay que mencionar, que son aspectos que se limitan a la vivienda. Retomando el concepto de habitabilidad, diversas condiciones que no han sido medidas por instituciones públicas, los residentes se encuentran inconformes, por ejemplo en lo que se refiere a equipamiento, espacio público y localización.

Una de las características que presentan estos conjuntos habitacionales, de manera particular Santa Teresa y Paseos de San Juan, es que se localizan en la periferia norte de sus respectivos municipios y no cuentan con suficientes escuelas, hospitales, supermercados y oferta de empleos, sin mencionar centros de entretenimiento (teatro, cine, centros culturales, etc.).

Según el Libro Quinto del Código Administrativo del Estado de México denominado "Del Ordenamiento Territorial de los Asentamientos Humanos y del Desarrollo Urbano de los Centros de Población", que es la normativa que regula la construcción de los conjuntos habitacionales en el estado de México, cada empresa inmobiliaria tiene la obligación de crear construcciones destinadas para escuelas de educación básica ${ }^{12}$. Sin embargo, a pesar de que hay escuelas, muchos

En conjuntos urbanos habitacionales de tipo interés social y popular, por cada mil viviendas previstas se debe construir un Jardín de niños de tres aulas, una escuela primaria o secundaria de doce aulas (llama la atención que puede ser una escuela primaria o una escuela secundaria). Además, se menciona que cuando se trate de conjuntos urbanos de más de cuatro mil viviendas, o cuando la Secretaría de Desarrollo Urbano y Metropolitano lo determine, considerando el equipamiento existente en la zona, se podrán sustituir parte de estos equipamientos por centros de educación media superior y superior u otros compatibles a la educación (Ver Gaceta, 2002). Sin embargo, en ninguno de estos conjuntos habitacionales hay una escuela preparatoria o universidad, a pesar de que los tres rebasan las 4,000 viviendas. 
residentes prefieren ir a otras escuelas, incluso de otros municipios, debido a que las condiciones físicas son muy malas, hay mucha inseguridad dentro de los conjuntos habitacionales e incluso no cuentan con maestros; "hay una sola secundaria, la cual no..., desde el prestigio que se tiene de los maestros de esa escuela, es muy, muy bajo. Los que tenemos hijos en secundaria tenemos que salir fuera." (Residente de Paseos de San Juan, Zumpango).

Respecto a centros de salud las condiciones son similares. Los hospitales cercanos no cuentan con el personal necesario e incluso ni con medicamentos: "Uuh, estamos de la chingada, de que chingados sirve la cruz roja si no hay doctores, pinche hospital que hicieron allá [se refiere al Hospital General] no sirve de ni madres porque tampoco hay doctores" (Residente de Santa Teresa, Huehuetoca). Por lo cual, mencionan los residentes que deben trasladarse a hospitales de municipios como Cuautitlán (a $42 \mathrm{~km}$ ) o Coacalco (a 28 km); "las urgencias están en Coacalco, cuando llegas ya moriste".

"¿Aquí? Un centro médico, un hospital estatal, nada de que hospitalitos de que aquí te atiendo y te vas a urgencias hasta por allá, no, no, no, aquí que tenga todo, es lo que se requiere ¿por qué? porque hay mucha gente, ahora te imaginas si tú vas a consulta a Cuautitlán, los pertenecientes al lado de Cuautitlán, los que son de aquel lado..., imagínate tú, una urgencia ¿a dónde te metes? [...] Ahora, por ejemplo, la situación de una embarazada, ¿a dónde corres? ¿A dónde van? Tienes dinero para pagar 9 mil o 10 mil pesos para aliviarte, que padre ¿y sino? O sea, te enfermas, una urgencia, aquí no hay, ¿buscas un doctor? no hay, ni particular." (Residente de Santa Teresa, Huehuetoca).

Además del insuficiente equipamiento, tanto escuelas como centros de salud que forman parte de dos derechos humanos fundamentales, las carencias de estos conjuntos habitacionales se aprecian en espacios comerciales, no necesariamente en tiendas de grandes superficies, pues la empresa Wall-Mart tiene presencia en los tres municipios, pero el comercio de proximidad, en donde se tejen relaciones sociales suele ser insuficiente. Esta situación repercute en poca convivencia entre vecinos. Aunado a ello, la falta de mantenimiento de espacios públicos, como áreas verdes o centros deportivos, incide en esta fragmentación social: "...los parques ya no esta cuidados. Y están todos descuidados, cuando yo me vine aquí, nos dijeron que esta área iba a estar siempre podada y la verdad aquí está pésimo, pero si te das una vuelta para allá atrás, está horrible. Hay muchos como que iban a ser mercaditos, pero quedaron ya abandonados. Están horribles ya." (Residente de Paseos de San Juan, Zumpango). Esta cita refleja parte de la problemática que se vive al interior de estos conjuntos habitacionales, donde no hay condiciones adecuadas para la convivencia entre los residentes, elemento central para el concepto de habitabilidad que proporciona Capron (2017).

El insuficiente equipamiento así como la lejanía de los conjuntos habitacionales a lugares de trabajo, incide en que los residentes invierten tiempo y dinero en su movilidad. Trayectos hacia los lugares de empleo, que se concentran en los municipios cercanos a la Ciudad de México y en la Alcaldías de la ciudad, pueden llegar a realizar entre 2 y 2 horas y media por trayecto. Mientras que en cuestión del costo de transporte suele representar hasta el 30\% del sueldo. Aunado a lo anterior, las malas condiciones de los autobuses así como las insuficientes rutas son un problema constante. 
Además de los problemas en cuestiones físicas de la vivienda, en los servicios básicos, en el insuficiente equipamiento, deterioro de espacios públicos y localización lejana, uno de los principales problemas es la inseguridad. Todos estos elementos han repercutido en que los propietarios decidan alquilar su vivienda e irse a alquilar a otra zona de la ciudad, e incluso muchos han abandonado su vivienda. El abandono de vivienda, como se había mencionado, es muy elevado en estos municipios que forman parte del estado de México, contribuyendo a que sea el estado que se encuentra en el 4 to lugar a nivel nacional.

\section{Reflexiones}

Las políticas de liberalización de suelo, las atribuciones de los gobiernos locales y la promoción privada de vivienda, todos mecanismos introducidos en la lógica de libre mercado, están dando como resultado que el mercado formal de vivienda social se localice en periferias urbanas con escaso desarrollo en infraestructura y equipamiento urbano.

La dinámica inmobiliaria, que ha caracterizado la expansión y segregación de la periferia norte de la ZMVM, ha sido posible por el fuerte subsidio a la demanda, de manera particular al sistema público de crédito hipotecario, el cual ha estimulado la oferta privada en suelo con localización lejana respecto a zonas de oferta de empleo, así como con escaso desarrollo urbano, es decir, deficiente equipamiento, infraestructura y servicios públicos. Dicha dinámica está reproduciendo diversas problemáticas de habitabilidad. Las características físicas e internas de las viviendas no responden a las necesidades básicas de las familias, sino a un modelo de producción en serie que, junto a factores como la localización lejana, buscan reducir costos de producción para las empresas inmobiliarias.

Las mediciones elaboradas por las instituciones públicas subrayan el mejoramiento en los espacios de las viviendas y en la cobertura de los servicios básicos, lo que podría ser nombrado como el rostro humano del neoliberalismo (Hidalgo, Alvarado y Rivas, 2017) en donde se destacan los "beneficios" de la política de vivienda; no obstante, más allá de contar con acceso o aparente cobertura, según las condiciones reflejadas a partir de la entrevistas realizadas a los residentes contrasta mucho con el discurso oficial. Aunado a lo anterior, las condiciones "externas" de la vivienda, es decir, aquellas que remiten al concepto de habitabilidad, que no son referidas en las mediciones realizadas por instituciones públicas, se reproducen diversas problemáticas, lo que ha repercutido en el aumento de la vivienda abandonada.

Mientras el Estado mantenga una política de vivienda apoyando la oferta mediante los subsidios a la demanda, sin regular la localización y características de la vivienda, el sector privado accederá al suelo más barato, localizado en la periferia de las grandes ciudades, el cual tiene escasa inversión en infraestructura y equipamiento urbano. De esta manera, se aprecia un evidente fracaso en materia de desarrollo urbano y una expansión en la segregación residencial, y un éxito en la dinámica del mercado inmobiliario apoyado por los ONAVIS y justificado por las mediciones de instituciones públicas.

Es importante destacar, más allá de la información cuantitativa en las que se basan las mediciones oficiales sobre las condiciones de habitabilidad, la pertinencia de los trabajos empíricos, 
lo cual nos permite explorar condiciones que no se aprecian en los datos estadísticos. Retomando la complejidad del concepto de habitabilidad (Capron, 2017; Coulomb, 2006), son los residentes quienes expresan tanto las condiciones físicas como los servicios, equipamiento y las relaciones sociales que se desarrollan en los conjuntos habitacionales. Por lo anterior, resulta pertinente cuestionarse a qué responde la necesidad de generar estas mediciones por parte de instituciones públicas, ¿se busca justificar la dinámica inmobiliaria? ¿establecer el avance de la política de vivienda a nivel internacional? Lo cierto es que lo que ha representado un éxito en materia económica para las empresas inmobiliarias, ha significado un fracaso en términos sociales.

\section{Bibliografía}

ARCAS, J; PAGĖS, A. y CASALS, M. El futuro del Hábitat: repensando la habitabilidad desde la sostenibilidad. El caso español. INVI, 2011, vol. 26. núm. 72, pp. 65 - 93.

CABRERA, D. Y GUILLÉN, M. La problemática del abandono de la vivienda de interés social en las ciudades globales, una mirada desde sus habitantes, en CADENA, J., AGUILAR, M. Y VÁZQUEZ, D. (coords). Las ciencias sociales y la agenda nacional. Reflexiones y propuestas desde las Ciencias Sociales. México: COMECSO, 2018, p. 313 - 329.

CAPRON, G. Cierres de calles: un desafío para la habitabilidad de la ciudad. Espacialidades, 2017, vol. 7, núm. 1, p.126-145.

CASTILLO, O. Y ALEJANDRE, G. La habitabilidad en la construcción del espacio: el caso de La Trinidad, Zumpango. Quivera, 2012, vol.14, No 2012-2, p. 49-72, Universidad Autónoma del Estado de México, Toluca, México.

CEBALLOS, O. Política habitacional y calidad de la vivienda. Reflexiones sobre la habitabilidad de la vivienda de bajo costo en Bogotá. Revista Bitácora Urbano Territorial, 2006, vol. 1, No 10, p. 148157, Universidad Nacional de Colombia, Colombia.

CÓDIGO DE EDIFICACIÓN DE VIVIENDA. CONAVI. 2010. Disponible en https://www.gob.mx/cms/ uploads/attachment/file/85460/Codigo_de_Edificacion_de_Vivienda.pdf. Consultado 15 de enero de 2019.

CONAVI. Programa Nacional de Vivienda 2007- 2012: hacia un desarrollo habitacional sustentable. Disponible en internet: https://www.cmic.org.mx/comisiones/sectoriales/vivienda/doc.index/ PNV_2007-2012.pdf

CONEVAL. Carencia en el acceso a los servicios básicos en la vivienda. Disponible en: https:// www.coneval.org.mx/rw/resource/coneval/med_pobreza/Servicios_basicos_de_la_vivienda_Censo_2010/Carencia_servicios_basicos_vivienda_2010.pdf. Consultado 10 de diciembre de 2018.

CONEVAL. Consejo Nacional de Evaluación de la Política de Desarrollo Social. Carencia en la calidad y espacios de la vivienda. Disponible en: https://www.coneval.org.mx/rw/resource/coneval/ 
med_pobreza/Calidad_y_espacios_en_la_vivienda_Censo_2010/Carencia_en_la_calidad_y_espacios_en_la_vivienda_2010.pdf. Consultado 18 de diciembre de 2018.

CONNOLLY, P. ¿Política de vivienda o política de construcción?, en CESOP, La vivienda en México. Construyendo análisis y propuestas. México: Centro de Estudios Sociales y de Opinión Pública, Cámara de Diputados/LIX Legislatura, 2006, p. 119 - 134.

COULOMB, R. La articulación entre política habitacional y desarrollo urbano nacional: una necesidad no atendida, en La vivienda en México: construyendo análisis y propuestas México: Centro de Estudios Sociales y de Opinión Pública, Cámara de Diputados / LIX Legislatura, 2006.

COULOMB, R Y SCHTEINGART, M. Entre el Estado y el mercado. La vivienda en el México de hoy. México: Cámara de Diputados LIX Legislatura-Universidad Autónoma Metropolitana, unidad Azcapotzalco-Porrúa, 2006.

DE MATTOS, C. Globalización y metamorfosis metropolitana en América Latina. De la ciudad a lo urbano generalizado. Revista de Geografía Norte Grande, 2010, vol. 47, p. 81-104.

EIBENSCHUTZ, R. y GOYA, C. (Coord.) Estudio de la integración urbana y social en la expansión reciente de las ciudades en México, 1996 - 2006: dimensiones, características y soluciones. México: Sedesol - Universidad Autónoma Metropolitana - Porrúa, 2009.

FIQUE, L. La política pública de vivienda en Colombia. Conflicto de ojetivos. Bitácora, 2008, vol. 2, p. 73 - 89.

GACETA DE GOBIERNO. Reglamento del Libro Quinto del Código Administrativo del Estado de México. 2002. Disponible en internet: https://legislacion.edomex.gob.mx/sites/legislacion.edomex. gob.mx/files/files/pdf/rgl/vig/rglvig107.pdf.

GAZMURI, P. Familia y habitabilidad en la vivienda. Aproximaciones metodológicas para su estudio desde una perspectiva sociológica. Arquitectura y Urbanismo, 2013, vol. 34, № 1, p. 32- 47.

GRAMSCH, J. Vivienda y transformaciones de habitabilidad en la provincia de Arauco. Urbano, 2006, vol. 9, No 14, p. 34-43.

HARVEY, D. From managerialism to entrepreneurialism: The transformation in urban governance in late capitalism. Geografiska Annaler. Series B, Human Geography, 1989, Vol. 71, No. 1, pp. 3-17,

HARVEY, D. Espacios del capital. Hacia una geografía crítica. España: Akal, 2001.

HIDALGO, R.; ALVARADO, V. y RIVAS, D. La espacialidad neoliberal de la producción de vivienda social en las áreas metropolitanas de Valparaíso y Santiago (1990-2014): ¿hacia la construcción idelógica de un rostro humano? Cadernos Metrópole, 2017, vol. 19, núm. 39, p. 513-535. 
INFONAVIT. Atlas del abandono de vivienda. México: Instituto del Fondo Nacional para la Vivienda de los Trabajadores, 2015.

INEGI. Censo de Población y Vivienda 1990, 2000 y 2010. Disponible: https://www.inegi.org.mx/ default.html.

INEGI. Encuesta Intercensal 2015. Disponible: https://www.inegi.org.mx/default.html.

KUNZ, I. Y SOLEDAD, A. Elementos de éxito en la producción social de la vivienda en México, Economía, Sociedad y Territorio, 2017, vol. xvii, núm. 55, p. 683-707.

LÁNDAZURI, A. M Y MERCADO, J. S. Algunos factores físicos y psicológicos relacionados con la habitabilidad interna de la vivienda. Medio Ambiente y Comportamiento Humano, 2004, vol. 5 No 1 y 2, p. $89-113$.

MARMOLEJO, C. y BATISTA N. Estructura urbana y segregación socioresidencial: un análisis para Maceió-Alagoas, Brasil. Papeles de población, 2011, vol. 17, núm. 70, p. 247-286.

MEJÍA, M. Habitabilidad en la vivienda social en edificios para población reasentada: El caso de Medellín, Colombia. EURE, 2012, vol. 38, núm. 114, p. 203-227.

MELLADO, R. La política de vivienda en las administraciones del Partido Acción Nacional: 2000 - 2012, en: ZICCARDI, A Y GONZÁLEZ, A. (coord.) Habitabilidad y política de vivienda en México. México: UNAM, p. 59 - 67. 2015.

MONKKONEN, P. La segregación residencial en el México urbano: niveles y patrones. EURE, 2012, vol. 38, núm. 114, p. 125-146.

MONTEJANO, J.; CAUDILLO, C. y CERVANTES, M. Vivienda de interés social, segregación residencial y accesibilidad: análisis de 121 conjuntos urbanos en el arco nororiente del Valle de México, 2001-2010. Estudios Demográficos y Urbanos, 2018, vol. 33, núm. 1 (97), pp. 187-224.

MORENO, S. La habitabilidad urbana como condición de calidad de vida. Palapa, 2008, vol. 3, No 2, p. 47-54, Universidad de Colima, México.

NASCIMENTO, M.; GÓMEZ, D. y SOUTO, R. Distribuição espacial e acesso a serviços públicos essenciais em políticas de habitação de interesse social. Revista de Políticas Públicas, 2017, vol. 21, núm. 1.

PEÑA, L. Y SANDOVAL, L. Ciudad Juárez: deterioro y abandono de vivienda. Ciudades, 2017, No 113, p. $28-36$.

REAL ESTATE MARKET \& LIFESTYLE. Política de vivienda, Propuestas 2006 - 2012. Disponible en: https://realestatemarket.com.mx/articulos/mercado-inmobiliario/industria/12187-politica-de-vivienda-propuestas-2006-2012. Consultado: 05 de enero de 2019. 
RINCÓN, M. Asentamientos de origen informal y habitabilidad. Lectura desde La justicia espacial. Bitácora, 2018, vol. 28, núm. 3, p. $39-46$.

ROBERTS, B. Globalization and Latin American cities. International Journal of Urban and Regional Research, 2005, vol. 29, No 1, p. $110-123$.

SABATINI, F.; CÁCERES, G. y CERDA, J. Segregación residencial en las principales ciudades chilenas: Tendencias de las tres últimas décadas y posibles cursos de acción. EURE, 2001, vol. 27, núm. 82 , p. 21-42.

SALAS, J. Tugurización y necesidades de habitabilidad básica, rémoras a la cohesión social en Latinoamérica. Pensamiento Iberoamericano, 2008, no. 1, p. 201 - 230.

SALINAS L. Política de vivienda y gestión metropolitana en la expansión de la periferia de la ZMCM. Cuadernos Geográficos, 2016, vol. 55, №. 2, p. $217-237$.

SECRETARÍA DE GOBERNACIÓN. Constitución Política de los Estados Unidos Mexicanos, 2014. Disponible en internet: http://www.gobernacion.gob.mx.

SECRETARÍA DE DESARROLLO URBANO Y METROPOLITANO DEL ESTADO DE MÉXICO. Conjuntos habitacionales autorizados. Datos recuperados del año 2000 a 2015.

SISTEMA NACIONAL DE INFORMACIÓN MUNICIPAL. Datos recuperados del año 1990, 2000 y 2010.

TOCORNAL, X.; TAPIA, R. y CARVAJAL, Y. Delincuencia y violencia en entornos residenciales de Santiago de Chile. Revista de Geografía Norte Grande, 2014, vol. 57, p. 83-101.

WARD, P. México megaciudad: desarrollo y política, 1970 - 2002, El Colegio Mexiquense, México, 2004.

ZICCARDI, A. Como viven los mexicanos, Análisis regional de las condiciones de habitabilidad de la vivienda. Encuesta nacional sobre las Condiciones de Habitabilidad de la Vivienda. UNAM, Instituto de Investigaciones Jurídicas, 2015.

ZULAICA, L Y CELEMÍN J. Análisis territorial de las condiciones de habitabilidad en el periurbano de la ciudad de Mar del Plata (Argentina), a partir de la construcción de un índice y de la aplicación de métodos de asociación espacial. Revista de Geografía Norte Grande, 2008, no. 41. p. 121 - 146.

ZUNINO, H. e HIDALGO, R. Las políticas de vivienda en el Chile neoliberal: efectos socioespaciales y relaciones de poder en el caso del Área Metropolitana de Valparaíso y su periferia, en HIDALGO, R.; DE MATTOS, C. y ARENAS, F. (editores) Chile: del país urbano al país metropolitano, Santiago de Chile: Serie GEOlibros, pp. 223 - 239. 2009. 
